\title{
Life-Threatening Atypical Case of Acute Generalized Exanthematous Pustulosis
}

\author{
Azadeh Tajmir-Riahi ${ }^{a}$ Petra Wörl ${ }^{a}$ Thomas Harrer ${ }^{b}$ Stefan Schliep ${ }^{a}$ \\ Gerold Schuler $^{\mathrm{a}}$ Miklos Simon ${ }^{\mathrm{a}}$ \\ Departments of a Dermatology and ${ }^{\mathrm{b}}$ Internal Medicine 3, University Hospital Erlangen, Friedrich-Alexander \\ University Erlangen-Nürnberg, Erlangen, Germany
}

\section{Established Facts}

- Acute generalized exanthematous pustulosis (AGEP) generally has a modest course.

- Patients with poor health or the elderly are prone to developing a severe course.

\section{Novel Insights}

- A young, healthy subject might develop an atypical AGEP, in this case leading to a critical internal status with systemic inflammatory response syndrome and treatment at an intensive care unit.

\section{Keywords}

Acute generalized exanthematous pustulosis - Systemic involvement · Systemic antibiotics · Drug eruption . Intensive care unit - Systemic inflammatory response syndrome

\begin{abstract}
Antibiotics are known to cause severe cutaneous adverse reactions, such as the rare acute generalized exanthematous pustulosis (AGEP). Unlike Stevens-Johnson syndrome or toxic epidermal necrolysis, AGEP is rarely life-threatening. Systemic involvement is not typical, and if present usually coincides with a mild elevation of the hepatic enzymes and a decrease in renal function. Hence, AGEP is known to have a good prognosis and to be life-threatening only in elderly patients or patients with chronic diseases. Herein, we report a case of AGEP in a young healthy male leading to systemic
\end{abstract}

(C) 2017 S. Karger AG, Basel

\section{KARGER}

E-Mail karger@karger.com

www.karger.com/iaa inflammatory response syndrome and to treatment in an intensive care unit after being treated with 5 different antibiotics. Initial symptoms were not indicative for AGEP and the patient's course of disease led promptly to critical cardiorespiratory symptoms and systemic inflammatory response syndrome. We assume that the administration of the 5 different antibiotics resulted in type IV allergy as well as secondary infection with Enterococcus faecium and Staphylococcus aureus, while the underlying periodontitis also contributed to the severity of this case.

(c) 2017 S. Karger AG, Basel

\section{Introduction}

Severe cutaneous adverse reactions (SCARs) are wellknown side effects of antibiotics such as $\beta$-lactams, cephalosporines, quinolone antibiotics, and macrolides [1-3]. 


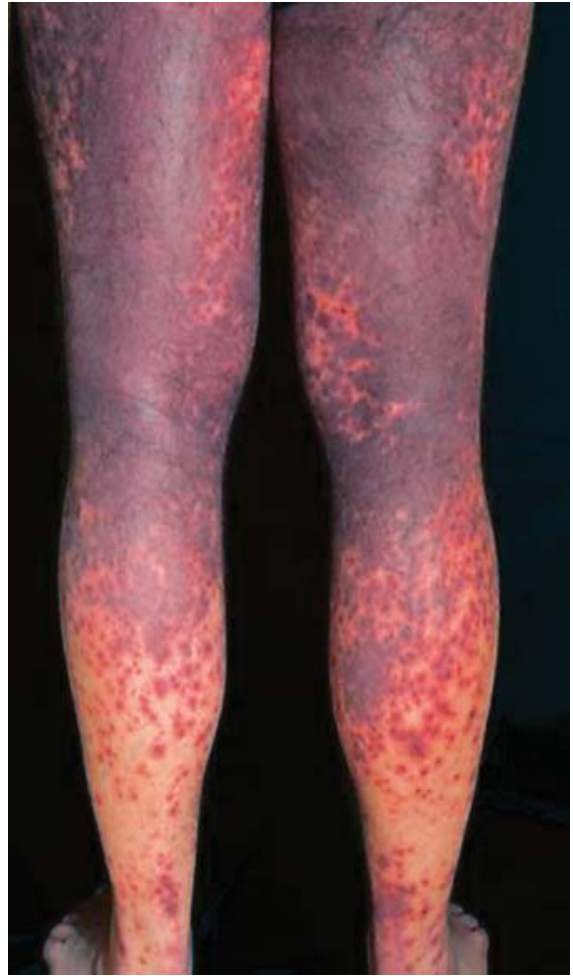

Fig. 1. Target lesion-like macules.

Acute generalized exanthematous pustulosis (AGEP) is a T-cell CD4+ and CD8+ mediated delayed-type hypersensitivity reaction with elevated IL-4, IL-5, and IL-8 expression [3-6], as well as IL-17A/F $/ \mathrm{F}^{+}$in the subcorneal pustules, in the epidermis near the pustules, and in the upper dermis [7] attributed to drugs, viral infections, or mercury [8].

The typical clinical features of AGEP are diffuse erythema with pustular cutaneous eruptions, acute fever, and leucocytosis occurring after the administration of the causative drug $[2,9]$. Systemic involvement with internal organ dysfunction is usually not expected and has rarely been reported [10]. Here, we report a case of AGEP validated with a EuroSCAR study group score, with systemic inflammatory response syndrome in a healthy young man.

\section{Case Report}

A 30-year-old Caucasian male presented in an external clinic with an erythema of the face. He had previously received amoxicillin perioral for the treatment of periodontitis. Subsequently, 1 day later, he developed a macular erythema of the face, and the antibiotic therapy

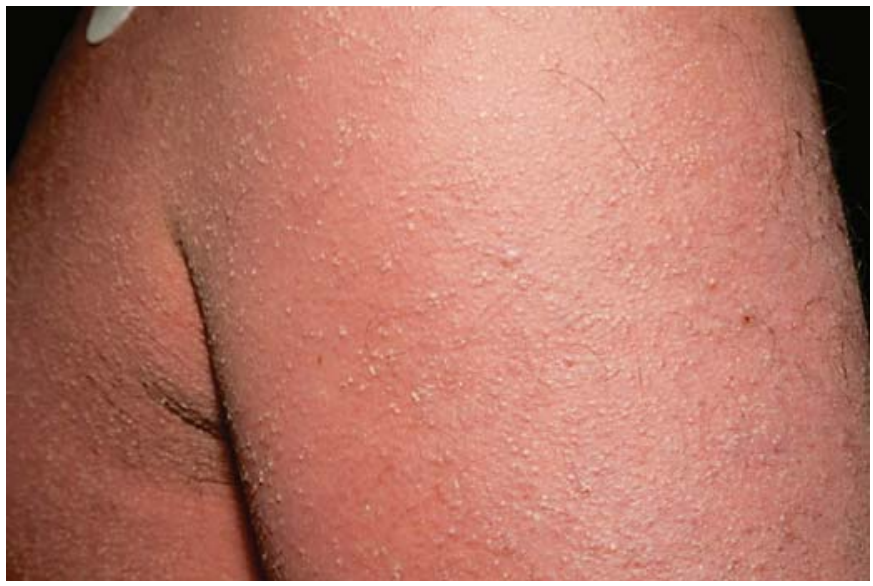

Fig. 2. Hundreds of pustules on erythematous and oedematous skin of the trunk.

was changed to ciprofloxacin $1 \mathrm{~g}$ /day for 4 days. On the 4 th day of treatment, he developed fever, chills, and erythema of the groin, while the erythema of the face subsided. Two days later, he was hospitalized with fever, chills, and a suspected infection. He received ampicillin/sulbactam and benzylpenicillin intravenously and subsequently the erythema spread to the rest of his skin, accompanied by fever over $39^{\circ} \mathrm{C}$ and pustulosis on the trunk and limbs. In addition, he developed conjunctivitis, weeping facial erosions, including the lips, erosions on the neck, and oral mucosa, and widespread erythema of the genitals with exfoliation. A diagnosis of Stevens-Johnson syndrome (SJS)/toxic epidermal necrolysis (TEN) due to ciprofloxacin was made and the patient was transferred to our hospital.

He had neither a significant past medical history, nor known drug allergies, and was not taking any other medications. His medical history was only positive for atopic dermatitis in the past and type I sensitization to pollen.

Upon physical examination, we noticed erythema of the trunk and legs with livid target lesion-like macules (Fig. 1). Multiple, non-follicular-bound small pustules appeared on the 7th day on the trunk (Fig. 2). The patient also developed severe involvement of the mucous membranes with erythema and erosions of the oral mucosa (Fig. 3) with accompanying impaired ingestions, conjunctivitis, as well as an anal erythema and exfoliation of the mucous membrane of the genital region.

Cultures from the pustules showed Enterococcus faecium, Propionibacterium granulosum, and $P$. acnes. Skin culture and a nasal swab showed massive Staphylococcus aureus.

Infectious work-up included HIV and hepatitis B/C serology, both of which turned out to be negative. Epstein-Barr virus serology showed a past infection.

The histologic examination showed several subcorneal pustules and exocytosis of neutrophil granulocytes with discrete spongiosis in the epidermis below. No vacuolar changes at the dermoepidermal interface were observed. In the upper dermis, a mixed inflammatory infiltrate of neutrophils, lymphocytes, and prominent eosinophils was present. The biopsy was assessed as AGEP. TEN was ruled out (Fig. 4) and immunofluorescence investigations were negative.

Atypical Case of AGEP

Int Arch Allergy Immunol 2017;174:108-111 


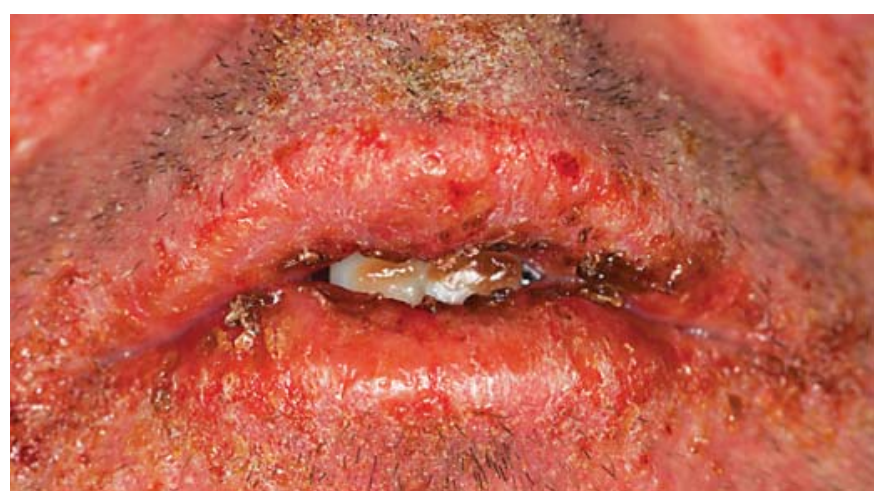

Fig. 3. Erosions with superinfection affecting the lips.

After validation with a EuroSCAR score, the diagnosis of AGEP was provided. We commenced a systemic glucocorticosteroid therapy of prednisolone $250 \mathrm{mg}$ i.v., which we then tapered to $1 \mathrm{mg} / \mathrm{kg}$ body weight. Since procalcitonin was elevated to $19.3 \mathrm{ng} /$ $\mathrm{mL}$ (normal range $<0.5 \mathrm{ng} / \mathrm{mL}$ ) and the patient had sustained signs of inflammation, fever $>40^{\circ} \mathrm{C}$, CRP $208.5 \mathrm{mg} / \mathrm{L}(<5 \mathrm{mg} / \mathrm{L})$, and neutrophilic granulocytes were increased to $24.1 \times 10^{3} / \mu \mathrm{L}$, we began calculated antibiotic treatment with clindamycin, meropenem, and vancomycin. Local therapy included glucocorticosteroid class III creams and antiseptic solutions.

The patient's clinical condition deteriorated and further workup was performed. X-ray of the lung showed signs of hypervolemia and mild diuretic therapy with i.v. furosemide was initiated. Mild proteinuria and oliguria were noted and a blood draw showed reduced total protein and elevation of liver enzymes, so we applied human albumin i.v. In addition, the patient developed hypotension and forced volume therapy was initiated. He subsequently developed critical cardiorespiratory symptoms with dyspnoea, tachypnoea, tachycardia, peripheral oedema, and anasarca. Due to suspected sepsis, we transferred him to the intensive care unit. Intensified diuresis was initiated and the patient lost $16 \mathrm{~kg}$ of weight. Antibiotic therapy was continued and the patient's cardiorespiratory symptoms stabilized. After 4 days, the antibiotics were stopped as blood cultures were negative except for S. epidermidis, which might have been caused by contamination. Prednisolone was tapered and stopped on the 12th day. We dismissed the patient on the 12th day with subsided cardiorespiratory symptoms and a significant improvement of the skin.

Four months later, a patch test was performed and revealed a positive late-type reaction to penicillins, including clemizole penicillin, phenoxymethylpenicillin, ampicillin, amoxicillin, and flucloxacillin. Prick testing was positive for ciprofloxacin, moxifloxacin, amoxicillin, penicillin, ampicillin, amoxicillin/clavulansäure, ceftriaxone, flucloxacillin, and phenoxymethylpenicillin. To avoid $\beta$-lactam antibiotics, gyrase inhibitors were recommended. Intradermal testing of minor and major determinants of penicillin were positive for penicillin $\mathrm{G}$ and ampicillin.

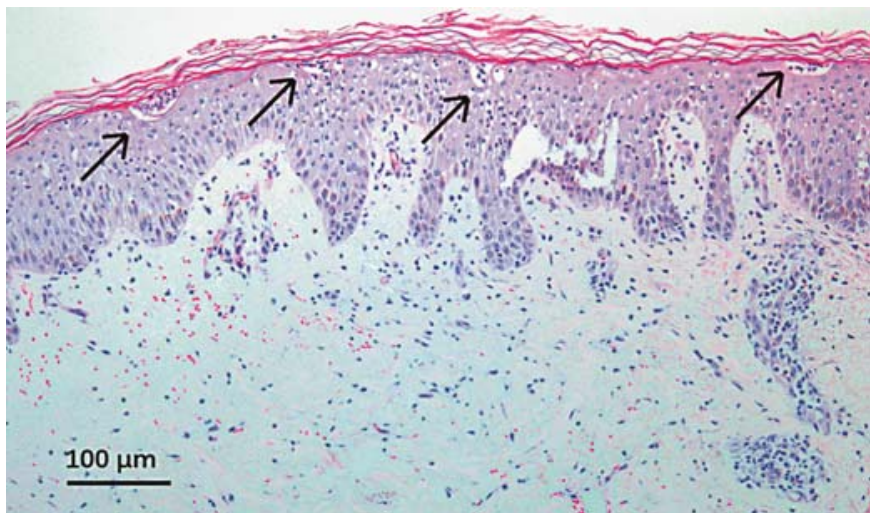

Fig. 4. Histologic presentation of the trunk showing exocytosis of neutrophils and subcorneal pustules (arrows). The upper dermis shows an inflammatory infiltrate of neutrophils, eosinophils, and lymphocytes.

\section{Discussion}

Our case demonstrates a rare case of life-threatening AGEP with atypical symptoms in a young healthy male. AGEP is known to be self-limiting without treatment in 5-7 days after discontinuation of the culprit drug [11], with a mortality rate of $1-2 \%$ caused by superinfection and a pre-existing compromised immune state [11]. Allergological work-up with the culprit drug is often positive in AGEP compared to SJS/TEN [12]. Severe cases are only reported in elderly patients or patients with chronic diseases [13]. Since skin symptoms usually appear after primary sensitization of about 1-3 weeks, and they appear within a few hours to 3 days [13], it is unclear if only 1 of the antibiotics was the causative drug. Since the faster courses are associated with patients who were rechallenged with the same drug after an occurrence of AGEP [13], we assume amoxicillin or ciprofloxacin to be the culprit drugs.

We assume that the underlying type I/IV allergies to various $\beta$-lactam antibiotics and the prior administration of amoxicillin, ciprofloxacin, ampicillin/sulbactam, and benzylpenicillin, together with the secondary infection of the skin with E. faecium, $P$. granulosum, $P$. acnes, and $S$. aureus, led to severe life-threatening systemic involvement. Pre-existing bacterial infections, in this case caused by periodontitis, might have also been a promoting factor [9].

Our case demonstrates a situation where an antibioticsensitized person received multiple incompatible antibiotics. Atypical symptoms were mucous membrane in-
Tajmir-Riahi/Wörl/Harrer/Schliep/ Schuler/Simon 
volvement of 3 locations, whereas only $20 \%$ of AGEP cases are associated with mucous membrane involvement, most commonly enoral mucosa $[8,12]$. The patient also developed conjunctivitis, which to our knowledge has not previously been reported in AGEP. This is the second published case, to our knowledge, reporting involvement of the lung [14]. Systemic involvement, if present, results in elevated liver enzymes and impaired renal function $[14,15]$.

Due to the risk of developing systemic involvement in AGEP, we recommend a quick transfer to a department with special knowledge in SCARs, even in young and healthy patients. Since the skin reaction showed an atypical pattern with target lesions on the legs, exfoliating skin, and was initially misdiagnosed as SJS/TEN, specific expertise in cases with suspected SCARs is necessary. Although arguments for cases with overlap of SJS/TEN and AGEP appear in the literature, there are no assured facts considering the EuroSCAR and RegiSCAR diagnostic criteria $[12,13]$. Careful administration of antibiotics is recommended, especially when bacterial infection is not evident.

\section{Disclosure Statement}

No non-financial or financial conflicts of interest exist for any of the authors. The patient gave his consent to the publication of his case.

\section{References}

1 Hotz C, Valeyrie-Allanore L, Haddad C, Bouvresse S, Ortonne N, et al: Systemic involvement of acute generalized exanthematous pustulosis: a retrospective study on 58 patients. Br J Dermatol 2013;169:1223-1232.

2 Schneck J, Fagot JP, Sekula P, Sassolas B, Roujeau JC, et al: Effects of treatments on the mortality of Stevens-Johnson syndrome and toxic epidermal necrolysis: a retrospective study on patients included in the prospective EuroSCAR study. J Am Acad Dermatol 2008;58: 33-40.

3 Sideroff A, Halevy S, Bouwes Bavinck JN, Vaillant L, Roujeau JC: Acute generalized exanthematous pustulosis (AGEP) - a clinical reaction pattern. J Cutan Pathol 2001;28:113119.

4 Lin YV, Yang CH, Sindy H, Lin JY, Hui RCY, et al: Severe cutaneous adverse reactions related to systemic antibiotics. Clin Infect Dis 2014;58:1377-1358.

5 Otero Rivas MM, Sanchez Sambucety P, Garcia-Ruiz de Morales JM, Pérez Paredes G, Rodriguez Prieto MA: Acute generalized exanthematous pustulosis due to dextromethorphan. Dermatol Online J 2013;19:10.
6 Hammerbeck AA, Daniels NH, Callen JP: Ioversol-induced acute generalized exanthematous pustulosis: a case report. Arch Dermatol 2009;145:683-687.

7 Kakeda M, Schlapbach C, Danelon G, Tang MM, Cecchinato V: Innate immune cells express IL-17A/F in acute generalized exanthematous pustulosis and generalized pustular psoriasis. Arch Dermatol Res 2014;306: 933-938.

8 Sideroff A, Dunant A, Viboud C, Halevy S, Bouwes Bavinck JS, et al: Risk factors for acute generalized exanthematous pustulosis (AGEP): results of a multinational case-control study (EuroSCAR). Br J Dermatol 2007; 157:989-996.

9 Hagiya H, Kimura M, Miyamoto, Haruki Y, Otsuka F: Acute generalized exanthematous pustulosis caused by daptomycin in a critically iII burn victim. Intern Med 2014;53: 511-514.

10 Kumar R, Qureshi S, Jakus J, Palma-Diaz FM: A severe case of acute generalized exanthematous pustulosis (AGEP) in a child after the administration of amoxicillin-clavulanic acid: brief report. Pediatr Dermatol 2009;26:623625.
11 Kostopoulos TC, Krishna SM, Brinster NK, Ortega-Loayza AG: Acute generalized exanthematous pustulosis: atypical presentations and outcomes. J Eur Acad Dermatol Venereol 2015;29:209-214.

12 Van Hattem S, Beerthuizen GI, Kardaun SH: Severe flucloxacillin-induced acute generalized exanthematous pustulosis (AGEP), with toxic epidermal necrolysis (TEN)-like features: does overlap between AGEP und TEN exist? Clinical report and review of the literature. Br J Dermatol 2014;171:1539-1545.

13 Wöhrl S: Clinical work-up of adverse drug reactions. Expert Rev Dermatol 2007;2:217231.

14 Lesterhuis WJ, Tjioe M, Stumpenhausen GA, van Crevel R: Acute generalized exanthematous pustulosis mimicking septic shock. Am J Med 2005;116:574-575.

15 Bomarrito L, Zisa G, Delrosso G, Farinelli P, Galimberti M: A case of acute generalized exanthematous pustulosis due to amoxicillinclavulanate with multiple positivity to betalactam patch testing. Eur Ann Allergy Clin Immunol 2013;45:178-180. 